\title{
Huge Hydatid Cyst of the Right Ventricular Outflow Tract
}

\author{
"Behnam Shakerian, ${ }^{1,2}$ and Mohammad H. Mandegar
}

\begin{abstract}
Hydatid disease is a common health problem in sheep-farming countries such as Iran. The liver and lungs are the most common primary sites of hydatid cysts in humans. Cardiac involvement is an uncommon manifestation, and the right ventricle outflow tract (RVOT) is rarely involved. This is a case report of a 34-year-old man who presented to the Heart Clinic, Tehran, Iran, in 2019 with a history of dyspnoea and fatigue. Following an imaging study, the patient was diagnosed with an RVOT hydatid cyst. He underwent surgical resection of the cyst. The post-operative course was uneventful.
\end{abstract}

Keywords: Hydatid Cyst; Right Ventricular; Outflow Obstruction; Case Report; Iran.

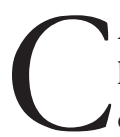

ARDIAC INVOLVEMENT IS UNCOMMON IN hydatid disease. The symptoms vary depending on the size and location of the cysts. Approximately $90 \%$ of the patients remain asymptomatic since hydatid cysts grow slowly; a period of 5-10 years may elapse before the lesions become large enough to cause cardiovascular symptoms. ${ }^{1}$ This is a case report of a hydatid cyst of the right ventricular outflow tract (RVOT) obstructing the pulmonary valve and an RVOT gradient. Cyst resection was performed using cardiopulmonary bypass. Cardiac hydatid disease is a rare condition that could be potentially life-threatening due to local and systemic complications. ${ }^{2}$ Surgery is the treatment of choice. ${ }^{3}$

\section{Case Report}

A 34-year-old male patient presented to the Heart Clinic, Tehran, Iran, in 2019 with a history of onemonth-long fatigue and 10-month-long progressive exertional dyspnoea. His past medical history was unremarkable and vital signs were stable. A physical examination revealed no specific findings except a systolic murmur in the pulmonary zone. Chest $\mathrm{X}$-ray, electrocardiogram and routine laboratory tests were normal. No cystic lesions were detected in thoracic, abdominal and brain scanning by computed tomography (CT). An enzyme-linked immunosorbent assay was negative for hydatic cysts. However, transthoracic echocardiography (TTE) showed a large $40 \times 40 \mathrm{~mm}$ hypoechoic mass without any internal debris in the RVOT, with an obstructive effect (pressure gradient $=48 \mathrm{mmHg}$ ) and a moderate right ventricle (RV) dilatation with mild dysfunction, representing a hydatid cyst [Figure 1]. Thoracic CT angiography showed a cystic lesion measuring about
$4.33 \times 4.20 \mathrm{~cm}$ in the RVOT, causing narrowing and obstruction of the RVOT, suggestive of a hydatid cyst [Figure 2].

Based on CT and echocardiography results and the high prevalence of hydatid cysts in Iran, the patient was scheduled for surgical excision. Median sternotomy was performed and the operative field was wrapped with towels moistened with hypertonic saline. After performing a cardiopulmonary bypass and inducing cardiac arrest, the whole content of the cyst was aspirated and a hypertonic saline solution was injected. A small incision was made into the cyst to remove its germinal layers [Figure 3].

The myocardial cavity was washed thoroughly with a hypertonic saline solution. Histological examination confirmed the diagnosis of a hydatid cyst. Postoperative TTE was normal, without any remnant of the cyst and normal pressure gradient of the RVOT. The postoperative period was uneventful and the patient was discharged from the hospital with oral albendazole. The patient gave his consent for publication of this case report and accompanying images.

\section{Discussion}

Cardiac hydatid disease, an exceedingly rare condition, was first described by Williams in $1836 .{ }^{4}$ It accounts for $0.5-2 \%$ of all cases of hydatid disease. ${ }^{5,6}$ Dogs are definitive hosts, while humans are accident hosts in their life cycle. After being eaten by the host, the parasite penetrates the mucosa of the duodenum and reaches the portal venous system. The liver acts as a filter for trapping the ova of Echinococcus granulosus. Some larvae may escape via the hepatic filter and may enter the systemic circulation leading to the heart. 


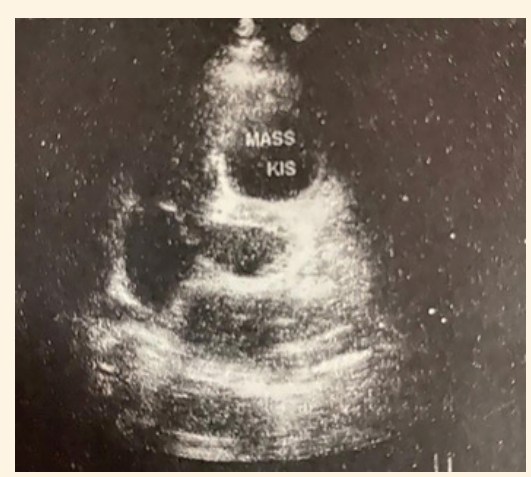

Figure 1: Transthoracic echocardiography view of the cardiac cyst in a 34-year-old male patient.

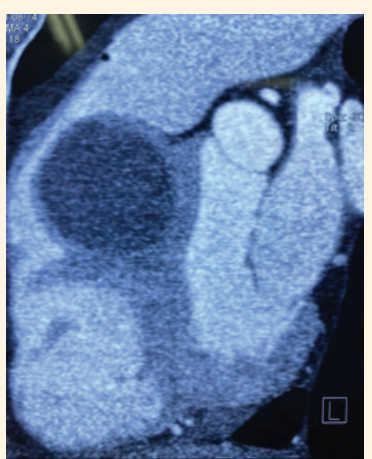

Figure 2: Computed tomography shows the hydatid cyst in a 34-year-old male patient.

After reaching the heart, the larvae become a mature cyst in about 1-5 years. Cardiac involvement may be seen through direct extension or haematogenous routes. $^{7}$ The larvae can reach the myocardium through coronary circulation. The left ventricle (LV) is most frequently involved, followed by the RV, interventricular septum, left atrium and right atrium. ${ }^{8}$

The patient may remain asymptomatic for many years or show symptoms depending on the location and size of the cysts, such as chest pain, dyspnoea, arrhythmia and anaphylactic reaction. Intracavitary rupture is more frequent in the RV compared to the LV and can cause pulmonary embolisation, pulmonary hypertension and death. Diagnosing cardiac hydatid cysts may be difficult due to the nonspecific symptoms and the crucial need for a high index of suspicion, especially in endemic areas. Patients with other systemic hydatid cysts should undergo echocardiography for identifying possible cardiac involvement. Echocardiography, CT and magnetic resonance imaging are the most important tools for diagnosis.

Serology for hydatid cysts is useful to confirm the diagnosis; however, studies suggest that the sensitivity of this is low, with false-negative results of up to $50 \%{ }^{9}$ Differential diagnosis includes intracardiac tumours, congenital cysts and ventricular aneurysms. ${ }^{10,11}$ Surgical excision is the treatment of choice, even in asymptomatic patients. As a rule, the heart should not be manipulated before applying a cross-clamp. ${ }^{8}$ Excision of myocardial hydatid cysts may be associated with complications such as damage to heart structures, cyst rupture, embolisation of the germinative membrane and contamination of surrounding structures. ${ }^{12}$

There are controversies about preoperative anthelmintic therapy in cardiac hydatid cysts. ${ }^{8,13}$ However, it is very important to bear in mind that anthelmintic therapy may lead to cyst death and destruction of its wall, resulting in cyst rupture. Therefore, germicides must not be administered before surgical removal. Anthelmintic therapy is advised if surgical treatment is refused or if the patient is inoperable. ${ }^{14}$

\section{Conclusion}

Isolated cardiac hydatid cysts are extremely rare. Early diagnosis and treatment are important to prevent lifethreatening complications. In tropical countries such as Iran, hydatid disease must always be kept in mind. To prevent recurrence after surgery, it is necessary to place patients on anthelmintic therapy.

\section{CONFLICT OF INTEREST}

The authors declare that there is no conflict of interest.

\section{AUTHORS' CONTRIBUTION}

BS and MHM contributed equally to the manuscript.
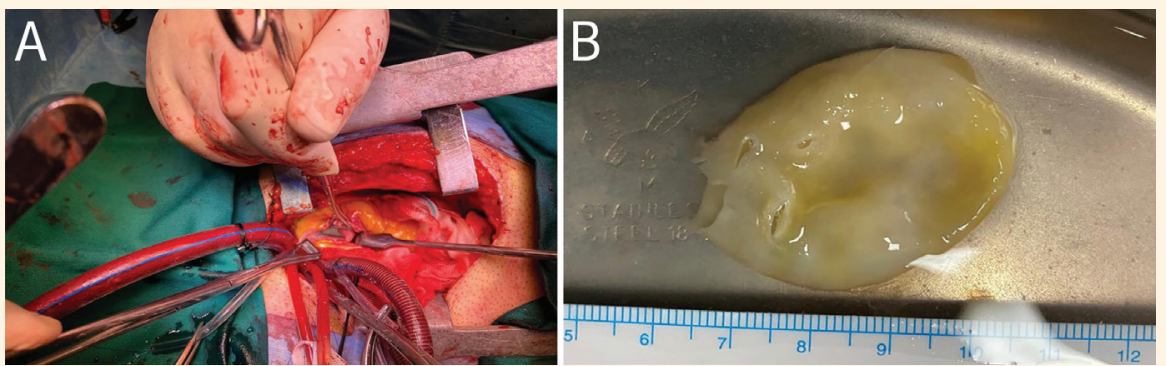

Figure 3: Intraoperative photographs showing (A) the cavity of the hydatid cyst in the right ventricle outflow tract and (B) the germinative membrane of the cyst. 
Both authors approved the final version of the manuscript.

\section{References}

1. Mehra S, Garga UC. Left ventricular myocardial hydatid cyst. Appl Radiol 2018; 47:36-8.

2. Mesrati MA, Mahjoub Y, Abdejlil NB, Boussaid M, Belhaj M, Limem H, et al. Case report: Sudden death related to unrecognized cardiac hydatid cyst. F1000Research 2020; 9:286. https://doi.org/10.12688/f1000research.23277.1.

3. Charfeddine S, Mallek S, Gueldiche M, Triki F, Jmaa HB, Frikha I, et al. A huge cardiac hydatid cyst: An unusual cause of chest pain revealing multivisceral hydatidosis in a young woman. I Saudi Heart Assoc 2015; 26:286-91. https://doi.org/10.1016/j. jsha.2015.04.003.

4. Yekeler I, Kocak H, Aydin NE, Basoğlu A, Okur A, Senocak H, et al. A case of cardiac hydatid cyst localized in the lungs bilaterally and on anterior wall of right ventricle. Thorac Cardiovasc Surg 1993; 41:261-3. https://doi.org/10.1055/s-2007-1013868.

5. Bogdanovic A, Radojkovic M, Jankovic TR, Pesic I, Radjenovic PT, Kovacevic P. Presentation of pericardial hydatid cyst as acute cardiac tamponade. Asian J Surg 2017; 40:175-7. https://doi. org/10.1016/j.asjsur.2013.10.001.

6. Agha R.A, Fowler A.J, Rammohan S, Barai I. Orgill DP, PROCESS Group. The PROCESS statement: Preferred reporting of case series in surgery. Int J Surg 2016; 36:319-23. https://doi. org/10.1016/j.ijsu.2016.10.025.
7. Acipayam M, Uncu H, Altinay L, Basdogan F, Gencaslan M, Özsöyler I. Cardiac hydatid cyst causing right ventricular outflow tract obstruction: Case report. Turkiye Klinikleri J Cardiovasc Sci 2013; 25:123-6.

8. Besir Y, Gucu A, Surer S, Rodoplu O, Melek M, Tetik O. Giant cardiac hydatid cyst in the interventricular septum protruding to right ventricular epicardium. Indian Heart J 2013; 65:81-3. https://doi.org/10.1016/j.ihj.2012.12.014

9. Tekbas EO, Tekbas G, Atilgan ZA, Islamoglu Y, Habib C, Yazici M. Left ventricle hydatid cyst mimicking acute coronary syndrome. J Infect Dev Ctries 2012; 6:579-83. https://doi. org/10.3855/jidc.1721.

10. Khosravi A, Taghipour H, Fanaei SA, Assar O, Ghyasy MS, Mirlohi SM. Heart hydatid cyst close to the left descending artery in a thirteen-year-old boy. Iran Red Crescent Med J 2014; 16: e15164. https://doi.org/10.5812/ircmj.15164.

11. Yildiz CE, Sinan UY, Yildiz A, Cetin G, Kucukoglu S. A case of isolated cardiac hydatid cyst that mimics lymphoproliferative malignancy. Echocardiography 2015; 32:1036-9. https://doi. org/10.1111/echo.12856

12. Moghul D, Hamidi, H. Incidental finding of cardiac hydatid cysts, report of two cases. BMC Med Imaging 2018; 18:1. https://doi.org/10.1186/s12880-018-0268-2.

13. Macedo AJ, Magalhães MP, Jalles TN, Bento L, Sampayo F, Lima M. Cardiac hydatid cyst in a child. Pediatr Cardiol 1997; 18:226-8. https://doi.org/10.1007/s002469900158.

14. Alshamlan RA, Almousa AM, Al Saeed MJ, Al-Dera HF, Alobaydun AM. Cardiac hydatid cyst successfully managed with Albendazole: A case report. Cureus 2019; 11: e6405. https://doi.org/10.7759/cureus.6405. 